\title{
Threshold-setting for Spectrum Sensing based on Statistical Information
}

\author{
Kenta Umebayashi, Member, IEEE, Kazuhiro Hayashi, and Janne J. Lehtomäki, Member, IEEE
}

\begin{abstract}
The use of prior information related to spectrum usage of primary users can enhance spectrum sensing performance. However, to the best of our knowledge, no analytical thresholdsetting method to achieve target sensing performance, such as detection probability, is reported in the literature. In this letter, a threshold-setting method based on approximate analysis for achieving target detection probability or false alarm probability is proposed. Numerical results confirm that the proposed thresholdsetting method is effective when the number of samples for the spectrum sensing is large or signal to noise power ratio is high.
\end{abstract}

Index Terms-Dynamic spectrum access, Spectrum sensing, Statistical information, Cognitive radio, Smart spectrum access.

\section{INTRODUCTION}

$\mathbf{I}$ $\mathrm{N}$ dynamic spectrum access (DSA), a secondary user (SU) can utilize spectrum licensed to a primary user (PU), while the spectrum is not occupied by the PU. Spectrum sensing [1][4] techniques have been investigated to detect the state of the spectrum part that is of interest. The state of spectrum can be either unoccupied $\left(H_{0}\right)$ or occupied $\left(H_{1}\right)$.

Energy detector (ED) is the simplest spectrum sensing method. It does not require any a priori information about PU signal [5]. However, the detection performance is not very good. One possible way to enhance spectrum sensing performance is to use statistical information of the PU's spectrum utilization as prior information [2]-[4]. In [2], spectrum utilization ratio is used to reduce detection error rate, which is defined as $\left(P_{\mathrm{FA}}=P\left(\hat{x}=1 \mid H_{0}\right)\right)+\left(P_{\mathrm{M}}=P\left(\hat{x}=0 \mid H_{1}\right)\right)$ where $P_{\mathrm{FA}}$ is false alarm probability, $P_{\mathrm{M}}$ is mis-detection probability, $\hat{x}$ denotes a spectrum sensing result, i.e., $\hat{x}=0$ and $\hat{x}=1$ indicates $H_{0}$ and $H_{1}$, respectively. Detection probability is defined by $\left(P_{\mathrm{D}}=P\left(\hat{x}=1 \mid H_{1}\right)\right)$ and obviously $P_{\mathrm{D}}=1-P_{\mathrm{M}}$. In [4], it is assumed that spectrum utilization by the PU follows a two states $\left(H_{0}\right.$ or $\left.H_{1}\right)$ discrete Markov model and transition probabilities are exploited in test statistics to enhance the detection performance. This method is denoted by Soft decision based Spectrum Sensing (SSS) in this letter and soft decision corresponds to probabilities of previous state $H_{i}$ ( $i \in\{0,1\}$ ). There is one important open issue in the spectrum sensing based on statistical information: analytical thresholdsetting to achieve target false alarm probability $\dot{P}_{\mathrm{FA}}$ or target detection probability $\dot{P}_{\mathrm{D}}$ has not been presented. Being able to reach a target false alarm or detection probability is the most important issue for spectrum sensing design. Especially, a satisfaction of $\dot{P}_{\mathrm{D}}$ is necessary for a protection of PU.

This research and development work was supported by the MICSCOPE \#165003006

K. Umebayashi, and K. Hayashi are with the Department of Electrical and Electronic Engineering, Tokyo University of Agriculture and Technology, Tokyo 183-8538, Japan

J. J. Lehtomaki is with the Centre for Wireless Communications (CWC), University of Oulu, 90570 Oulu, Finland.

Manuscript received Feb. 17, 2017; revised March 4, 2017
In this letter, threshold setting is proposed based on Ideal Spectrum Sensing (ISS), which assumes that the previous spectrum state is known. We obtain analytical expressions for $P_{\mathrm{D}}$ and $P_{\mathrm{FA}}$ as a function of threshold $\gamma$. In the analysis, we will show that the spectrum sensing can be converted to ED that selects threshold according to probability of $H_{1}$. Based on this investigation, a generalized form of analysis is shown. Specifically, this generalized analysis is applicable in not only in the two-state discrete Markov model but also spectrum usage models with more than two states. The assumption about the previous spectrum state in ISS is not practical. Therefore, we evaluate suitability of the proposed threshold setting under SSS, and Hard decision based Spectrum Sensing (HSS) where hard decision corresponds to previous spectrum sensing result.

\section{SPECTRUM SENSING MODEL}

A state of PU spectrum utilization in each time slot follows a two-state $\left(H_{0}\right.$ or $\left.H_{1}\right)$ discrete Markov model. Transition probability of the Markov model is denoted by $g_{k, j}(k, i \in\{0,1\})$, which indicates a probability that the state changes from $H_{k}$ in the current time slot to $H_{i}$ in the next time slot. The $n$th observed signal $(n=0,1, \cdots, N-1)$ by the $\mathrm{SU}$ in the $t$ th time slot is given by

$$
\begin{array}{ll}
H_{0}: & y_{t}(n)=w(n) \\
H_{1} & : \quad y_{t}(n)=s(n)+w(n),
\end{array}
$$

where the time duration of $N$ samples is assumed to be smaller than the time duration of one time slot, $s(n)$ denotes a deterministic (but unknown) complex PU signal, and $w(n)$ is complex additive white Gaussian noise (AWGN), i.e., $\mathcal{C N}\left(0, \sigma_{n}^{2}\right)$, where $\sigma_{n}^{2}$ is the variance. The effect of multi-path fading is not considered. The signal to noise power ratio is defined by $\nu=\sigma_{s}^{2} / \sigma_{n}^{2}$, where $\sigma_{s}^{2}$ denotes the power of $s(n)$. The output of ED at the $t$ th time slot is

$$
Y_{t}=\sum_{n=0}^{N-1}\left|y_{t}(n)\right|^{2}
$$

The statistical information-based spectrum sensing result in the $t$ th time slot is obtained by an estimated probability of $H_{0}$, $P\left(x_{t}=0\right)$, as [4]

$$
\hat{x}_{t}=\left\{\begin{array}{lll}
0 & \left(\text { if } P\left(x_{t}=0 \mid \mathbf{Y}_{0 \rightarrow t} ; \boldsymbol{\Phi}\right) \geq \gamma\right) & : H_{0} \\
1 & \text { (otherwise) } & : H_{1}
\end{array}\right.
$$

where $x_{t}$ denotes a state of spectrum utilization by PU at the $t$ th time slot and it can be either 0 or $1, \mathbf{Y}_{0 \rightarrow t}$ denotes a vector of outputs of $\mathrm{ED}$ from the 0th to the $t$ th time slots, and $\boldsymbol{\Phi}$ denotes a vector of parameters in terms of a priori information of PU signal and statistical information of PU spectrum usage. The state decision is obtained on the basis of thresholding the estimated probability with $\gamma$, where $0 \leq \gamma \leq$ 
1. The vector of parameters, $\boldsymbol{\Phi}$, consists of initial probabilities $\pi_{i}$, transition probabilities $g_{k, i}$, mean of observed ED outputs $\mu_{i}$, and variance of observed ED outputs $\sigma_{i}^{2}$, where the suffix $i$ indicates the state of spectrum $H_{i}$, i.e., [4]

$$
\mathbf{\Phi}=\left[\pi_{0}, \pi_{1}, g_{0,0}, g_{0,1}, g_{1,0}, g_{1,1}, \mu_{0}, \mu_{1}, \sigma_{0}^{2}, \sigma_{1}^{2}\right] \text {. }
$$

For estimating the parameters in $\boldsymbol{\Phi}$, the Baum-Welch algorithm [6] is applied in [4]. In SSS, estimated probability of $H_{i}$ in the $t$ th time slot is [4]

$$
\begin{aligned}
& P^{(S)}\left(x_{t}=i \mid \mathbf{Y}_{0 \rightarrow t} ; \mathbf{\Phi}\right) \\
& =\frac{1}{c_{t}}\left(\sum_{k=0}^{1} P\left(x_{t-1}=k \mid \mathbf{Y}_{0 \rightarrow t-1} ; \boldsymbol{\Phi}\right) \cdot g_{k, i}\right) \cdot p\left(Y_{t} \mid \mu_{i}, \sigma_{i}^{2}\right),
\end{aligned}
$$

where $c_{t}$ is the normalization coefficient

$c_{t}=\sum_{i=0}^{1} \sum_{k=0}^{1} P\left(x_{t-1}=k \mid \mathbf{Y}_{0 \rightarrow t-1} ; \boldsymbol{\Phi}\right) \cdot g_{k, i} \cdot p\left(Y_{t} \mid \mu_{i}, \sigma_{i}^{2}\right)$

and $p\left(Y_{t}\right)$ is the probability of density function (PDF) of $Y_{t}$. Equation (4) implies that the probability can be obtained in a recursive manner, while the probability for $x_{0}=i$ is given by

$$
P\left(x_{0}=i \mid Y_{0} ; \boldsymbol{\Phi}\right)=\frac{\pi_{i} p\left(Y_{0} \mid \mu_{i}, \sigma_{i}^{2}\right)}{\sum_{k=0}^{1} \pi_{k} p\left(Y_{0} \mid x_{t}=k ; \mu_{k}, \sigma_{k}^{2}\right)},
$$

where the initial probability $\pi_{i}$ indicates probability of $H_{i}$ in the 0th time slot. It may be available from a prior information by spectrum usage measurement [7], but if $\pi$ is not available, arbitrary value for $\pi$ may be used. In SSS, the soft decision corresponds to the estimated probability $P\left(x_{t-1}=k \mid \mathbf{Y}_{0 \rightarrow t-1} ; \boldsymbol{\Phi}\right)$ in (4).

In ISS, since the actual state $x_{t-1}$ is assumed to be known, the probability of $x_{t}=i$ is obtained by

$$
P^{(\mathrm{I})}\left(x_{t}=i \mid Y_{t} ; \boldsymbol{\Phi}, x_{t-1}\right)=g_{x_{t-1}, i} p\left(Y_{t} \mid \mu_{i}, \sigma_{i}^{2}\right) / c_{t}^{(\mathrm{I})},
$$

where $c_{t}^{(\mathrm{I})}=\sum_{i=0}^{1} g_{x_{t-1}, i} p\left(Y_{t} \mid \mu_{i}, \sigma_{i}^{2}\right)$. In HSS, the probability of $x_{t}=i$ is obtained by the hard decision, which is the previous sensing result $\hat{x}_{t-1}$, as

$$
P^{(\mathrm{P})}\left(x_{t}=i \mid Y_{t} ; \boldsymbol{\Phi}, \hat{x}_{t-1}\right)=g_{\hat{x}_{t-1}, i} p\left(Y_{t} \mid \mu_{i}, \sigma_{i}^{2}\right) / c_{t}^{(\mathrm{P})},
$$

where $c_{t}^{(\mathrm{P})}=\sum_{i=0}^{1} g_{\hat{x}_{t-1}, i} p\left(Y_{t} \mid \mu_{i}, \sigma_{i}^{2}\right)$. Decision result for ISS (or HSS) can be obtained by substituting $P^{(\mathrm{I})}$ (or $P^{(\mathrm{P})}$ ) into $P\left(x_{t}=0 \mid \mathbf{Y}_{0 \rightarrow t} ; \boldsymbol{\Phi}\right)$ in (3). In the spectrum sensing problem in (3), probability of $x_{t}=0$ is enough, but probability of $x_{t}=i$ is used in (4-8) for the sake of generality.

\section{ANALYSIS AND THRESHOLD-SETTING}

In ISS, the decision result would be $\hat{x}_{t}=0$, if the following inequality is satisfied:

$$
P^{(\mathrm{I})}\left(x_{t}=0 \mid Y_{t} ; \boldsymbol{\Phi}, x_{t-1}\right) \geq \gamma
$$

The PDF $p\left(Y_{t}\right)$ required in (7) is approximated by Gaussian distribution. By substituting (7) with Gaussian distribution into (9), we get

$$
\frac{\frac{g_{x_{t-1}, 0}}{\sqrt{2 \pi \sigma_{0}^{2}}} \exp \left(-\left(Y_{t}-\mu_{0}\right)^{2} /\left(2 \sigma_{0}^{2}\right)\right)}{\sum_{i=0}^{1} \frac{g_{x_{t-1}, i}}{\sqrt{2 \pi \sigma_{i}^{2}}} \exp \left(-\left(Y_{t}-\mu_{i}\right)^{2} /\left(2 \sigma_{i}^{2}\right)\right)} \geq \gamma
$$

and this equation can be considered as a quadratic inequality in terms of $Y_{t}$. Therefore, (10) can be expressed by

$$
a Y_{t}^{2}+2 b Y_{t}+c \geq 0,
$$

where $a, b$, and $c$ are the coefficients for the quadratic inequality and $a=\sigma_{0}^{2}-\sigma_{1}^{2}<0, b=\mu_{0} \sigma_{1}^{2}-\mu_{1} \sigma_{0}^{2}$, and

$$
c=\mu_{1}^{2} \sigma_{0}^{2}-\mu_{0}^{2} \sigma_{1}^{2}-2 \sigma_{0}^{2} \sigma_{1}^{2} \ln \left(\frac{\gamma g_{x_{t-1}, 1} \sigma_{0}}{(1-\gamma) g_{x_{t-1}, 0} \sigma_{1}}\right) .
$$

Statistics of $Y_{t}$ are given by $\mu_{0}=N \sigma_{n}^{2}, \sigma_{0}^{2}=N \sigma_{n}^{4}, \mu_{1}=$ $N \sigma_{n}^{2}\left(\sigma_{s}^{2}+1\right)$, and $\sigma_{1}^{2}=N \sigma_{n}^{4}\left(2 \sigma_{s}^{2}+1\right)$ [8]. Then, we obtain the following two inequalities for $\hat{x}_{t}=0$ :

$$
\mu_{0} / 2 \pm \sqrt{u\left(x_{t-1}\right)} /\left(2 \sigma_{s}^{2}\right) \gtrless Y_{t}
$$

where

$$
\begin{aligned}
u\left(x_{t-1}\right)= & N \sigma_{n}^{4} \sigma_{s}^{2}\left(2 \sigma_{s}^{2}+1\right) . \\
& \left(N \sigma_{s}^{2}-4 \ln \left(\frac{\gamma g_{x_{t-1}, 1}}{(1-\gamma) g_{x_{t-1}, 0} \sqrt{2 \sigma_{s}^{2}+1}}\right)\right) .
\end{aligned}
$$

In (12), only $\mu_{0} / 2+\sqrt{u\left(x_{t-1}\right)} /\left(2 \sigma_{s}^{2}\right)>Y_{t}$ provides proper decision. The other decision rule (if $\mu_{0} / 2-\sqrt{u\left(x_{t-1}\right)} /\left(2 \sigma_{s}^{2}\right)<$ $\left.Y_{t}, x_{t}=0\right)$ may be always satisfied under $H_{1}$ and this leads to $P_{\mathrm{D}} \simeq 0$. The decision rule $\left(\mu_{0} / 2+\sqrt{u\left(x_{t-1}\right)} /\left(2 \sigma_{s}^{2}\right)>Y_{t}\right)$ can be interpreted to ED as

$$
\hat{x}_{t}=\left\{\begin{array}{lll}
0 & \left(\text { if } Y_{t}<\gamma_{x_{t-1}}^{(\mathrm{ED})}\right) & : H_{0} \\
1 & \text { (otherwise) } & : H_{1}
\end{array}\right.
$$

where $\gamma_{x_{t-1}}^{(\mathrm{ED})}=N \sigma_{n}^{2} / 2+\sqrt{u\left(x_{t-1}\right)} /\left(2 \sigma_{s}^{2}\right)$ corresponds to threshold for ED. The threshold $\gamma_{x_{t-1}}^{(\mathrm{ED})}$ is selected according to the previous state $x_{t-1}$. A similar aspect can be confirmed in (7), in which the probability depends on the previous state $x_{t-1}$. Averaging the detection probability achieved by each thresholds $\gamma_{k}^{(\mathrm{ED})}$, the achievable detection probability with a given $\gamma$ is given as

$$
\begin{aligned}
P_{\mathrm{D}} & =\sum_{k=0}^{1} \frac{\operatorname{Pr}\left(H_{k}\right) \cdot g_{k, 1}}{\operatorname{Pr}\left(H_{0}\right) \cdot g_{0,1}+\operatorname{Pr}\left(H_{1}\right) \cdot g_{1,1}} \cdot Q\left(\frac{\gamma_{k}^{(\mathrm{ED})}-\mu_{1}}{\sqrt{\sigma_{1}^{2}}}\right) \\
& =\sum_{k=0}^{1} g_{1, k} \cdot \\
Q & \left(\frac{\sqrt{\sigma_{s}^{2}\left(N \sigma_{s}^{2}-4 \ln \left(\frac{\gamma g_{k, 1}}{(1-\gamma) g_{k, 0} \sqrt{\Lambda}}\right)\right)}-\sqrt{N \sigma_{s}^{4}(\Lambda)}}{2 \sigma_{s}^{2}}\right),
\end{aligned}
$$

where $Q(x)$ is the Q-function given by $Q(x)=$ $1 / \sqrt{2 \pi} \int_{x}^{\infty} \exp \left(-t^{2} / 2\right) d t$, and represents the detection probability with $\gamma_{k}^{(\mathrm{ED})}$, and $\Lambda=2 \sigma_{s}^{2}+1$. In fact, $P_{\mathrm{D}}$ is a 
monotonically increasing function of $\gamma$ and the region of $\gamma$ is limited to $0<\gamma<1$. Therefore, the threshold satisfying $\dot{P}_{\mathrm{D}}$ can be found according to (14) in a numerical manner. In addition, achieved false alarm probability is given as

$$
\begin{aligned}
P_{\mathrm{FA}} & =\sum_{k=0}^{1} \frac{\operatorname{Pr}\left(H_{k}\right) \cdot g_{k, 0}}{\operatorname{Pr}\left(H_{0}\right) \cdot g_{0,0}+\operatorname{Pr}\left(H_{1}\right) \cdot g_{1,0}} \cdot Q\left(\frac{\gamma_{k}^{(\mathrm{ED})}-\mu_{0}}{\sqrt{\sigma_{0}^{2}}}\right) \\
& =\sum_{k=0}^{1} g_{0, k} \cdot \\
Q & \left(\frac{\sqrt{\sigma_{s}^{2} \Lambda\left(N \sigma_{s}^{2}-4 \ln \left(\frac{\gamma g_{k, 1}}{(1-\gamma) g_{k, 0} \sqrt{\Lambda}}\right)\right)}-\sqrt{N \sigma_{s}^{4}}}{2 \sigma_{s}^{2}}\right)
\end{aligned}
$$

To facilitate the intuitive understanding of the improvement in the spectrum sensing due to statistical information, the reason for the improvement is given with an example of a two-state Markov model $\left(g_{0,0}=g_{1,1}=0.8\right)$ as follows. In the ED without statistical information, a single threshold satisfying $\dot{P}_{\mathrm{D}}$ is used and it can achieve a false alarm rate $\bar{P}_{\mathrm{FA}}$. The ED with statistical information in (13) utilizes the two thresholds. In case of $x_{t-1}=1$, the next state is also $x_{t}=1$ with $80 \%$ probability and the ED with statistical information sets the threshold $\gamma_{1}^{(\mathrm{ED})}$ to increase the detection probability to $\left(\dot{P}_{\mathrm{D}}+\Delta P_{\left.\mathrm{D} \mid \gamma_{1}^{(\mathrm{ED})}\right)}\right)$, while the false alarm probability also increases to $\left(\bar{P}_{\mathrm{FA}}+\Delta P_{\left.\mathrm{FA} \mid \gamma_{1}^{(\mathrm{ED})}\right)}\right.$, where $\Delta P_{\mathrm{D} \mid \gamma_{k}^{(\mathrm{ED})}}$ and $\Delta P_{\mathrm{FA} \mid \gamma_{1}^{(\mathrm{ED})}}$ indicates the variations of detection and false alarm probabilities from $\dot{P}_{\mathrm{D}}$ and $\bar{P}_{\mathrm{FA}}$ by using two thresholds, respectively. On the other hand, in case of $x_{t-1}=0$, the next state is $x_{t}=1$ with $20 \%$ probability. In this case, the threshold $\gamma_{0}^{(\mathrm{ED})}$ is set to decrease the false alarm rate $\left(\bar{P}_{\mathrm{FA}}-\Delta P_{\left.\mathrm{FA} \mid \gamma_{0}^{(\mathrm{ED})}\right)}\right)$, while the detection probability reduces

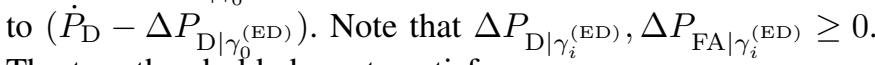
The two thresholds have to satisfy

$$
\dot{P}_{\mathrm{D}}=0.8\left(\dot{P}_{\mathrm{D}}+\Delta P_{\left.\mathrm{D} \mid \gamma_{1}^{(\mathrm{ED})}\right)}+0.2\left(\dot{P}_{\mathrm{D}}-\Delta P_{\left.\mathrm{D} \mid \gamma_{0}^{(\mathrm{ED})}\right)}\right)\right.
$$

In fact, there are multiple solutions $\left(\gamma_{1}^{(\mathrm{ED})}, \gamma_{0}^{(\mathrm{ED})}\right)$ satisfying $\dot{P}_{\mathrm{D}}$. The optimum solution is given by minimizing the false alarm probability given by

$$
P_{\mathrm{FA}}=0.2\left(\bar{P}_{\mathrm{FA}}+\Delta P_{\left.\mathrm{FA} \mid \gamma_{1}^{(\mathrm{ED})}\right)}\right)+0.8\left(\bar{P}_{\mathrm{FA}}-\Delta P_{\left.\mathrm{FA} \mid \gamma_{0}^{(\mathrm{ED})}\right)}\right)
$$

A gain in spectrum sensing is available if $0.2 \Delta P_{\mathrm{FA}, 1}-$ $0.8 \Delta P_{\mathrm{FA}, 0}<0$ while satisfying (16). When the gap of the above probabilities, 0.2 and 0.8 , which are determined by $g_{k, i}$, is large, high gain in detection performance may be achieved.

We generalize this ED with statistical information as follows. We define a set of states $S_{i}$ of PU spectrum usage as $\mathbf{S}=\left\{S_{i}\right\}$, in which the probability of $H_{1}$ is given by $\operatorname{Pr}\left(H_{1} \mid S_{i}\right)$. The probability for $S_{i}$ is denoted by $\operatorname{Pr}\left(S_{i}\right)$. This model is applicable in cases in which the probability of $H_{1}$ varies with $S_{i}$, such as the deterministic spectrum usage model [7], but is not limited to the discrete Markov model. Generalized spectrum sensing exploiting the statistical information $\mathbf{S}=\left\{S_{i}\right\}$ and $\operatorname{Pr}\left(H_{1} \mid S_{i}\right)$ selects proper threshold $\gamma_{i}$ for ED in response to the state $S_{i}$. Let $\gamma=\left\{\gamma_{i}\right\}$ denote a set of thresholds and the number of elements of $\mathbf{S}$ is $I$. In this case, the optimum threshold set minimizing $P_{\mathrm{FA}}$ is given by

$$
\begin{aligned}
& \gamma^{\mathrm{OPT}}=\arg \min _{\gamma} P_{\mathrm{FA}} \\
& =\arg \min _{\gamma} \frac{1}{c_{0}^{(G)}} \sum_{i=0}^{I-1} \operatorname{Pr}\left(H_{0} \mid S_{i}\right) \operatorname{Pr}\left(S_{i}\right) Q\left(\frac{\gamma_{i}-\mu_{0}}{\sqrt{\sigma_{0}^{2}}}\right),
\end{aligned}
$$

subject to

$$
\dot{P}_{\mathrm{D}}=\frac{1}{c_{1}^{(G)}} \sum_{\gamma_{i} \in \boldsymbol{\gamma}^{\mathrm{OPT}}} \operatorname{Pr}\left(H_{1} \mid S_{i}\right) \operatorname{Pr}\left(S_{i}\right) Q\left(\frac{\gamma_{i}-\mu_{1}}{\sqrt{\sigma_{1}^{2}}}\right),
$$

where $c_{k}^{(G)}=\sum_{i} \operatorname{Pr}\left(H_{k} \mid S_{i}\right) \operatorname{Pr}\left(S_{i}\right)$.

\section{NUMERICAL EVALUATIONS}

Common simulation parameters are as follows: $g_{0,0}=$ $g_{1,1}=0.8$ and ED stands for the conventional ED without statistical information. The prior information $\boldsymbol{\Phi}$ can be estimated by spectrum measurements and is available in SUs [4], [7]. We assume that $\boldsymbol{\Phi}$ is available without estimation error and this ideal assumption is reasonable when the estimation error is negligible. The analysis under the ideal assumption represents basis for analytical threshold setting under nonnegligible estimation error and this arises as an interesting issue for being investigated in future works.

We confirm the detection performance of spectrum sensing methods SSS, HSS, ISS, and ED by receiver operating characteristic (ROC) in Fig. 1 with $N=50$ and $N=100$, and $\nu=-5 \mathrm{~dB}$. The results are obtained by Monte Carlo simulations, but analytical result for ISS is also plotted. The detection performance of ISS is the best since it assumes that $x_{t-1}$ is known. The analytical result for ISS substantially coincides with the simulation result. In SSS, a probability of $x_{t-1}=0$ is estimated based on observed $t$ time slots. On the other hand, in HSS, the spectrum-sensing result from the previous time slot, $\hat{x}_{t-1}$, is used. Therefore, detection performance of SSS is better than that of HSS. The detection performance of ED is the poorest since the statistical information is not used. The differences in terms of spectrum sensing performances are due to the accuracy of the obtainable statistical information. It can be also confirmed that increasing $N$ can reduce the gaps among ISS, SSS, and HSS.

In Fig. 2, $P_{\mathrm{D}}$ as a function of $N$ in cases of $\nu=-5 \mathrm{~dB}$ and $\nu=-2 \mathrm{~dB}$ is evaluated by Monte Carlo simulations for SSS, HSS and ISS to confirm validity of the proposed threshold setting. Target detection probability is set to $\dot{P}_{\mathrm{D}}=0.9$. The threshold used in SSS and HSS are set based on the proposed threshold setting (14). The gap between $P_{\mathrm{D}}$ and $\dot{P}_{\mathrm{D}}$ is caused by the Gaussian approximation in (10). It can be confirmed that the gap can be reduced by increasing $N$.

In case of $\nu=-5 \mathrm{~dB}, P_{\mathrm{D}}$ of HSS in the region of $N<$ 30 is significantly high. The reason will be clarified with an investigation for Fig. 3 later. $P_{\mathrm{D}}$ of SSS in the region of $N<$ 200 still does not agree with $P_{\mathrm{D}}$ of ISS, but they coincide in the region where $N>300$. This is because in the region $N>300, P\left(x_{t-1}=i \mid \mathbf{Y}_{0 \rightarrow t-1} ; \boldsymbol{\Phi}\right) \approx 1$ for $x_{t-1}=i$ and this is eventually equivalent to ISS in this region. A comparison 


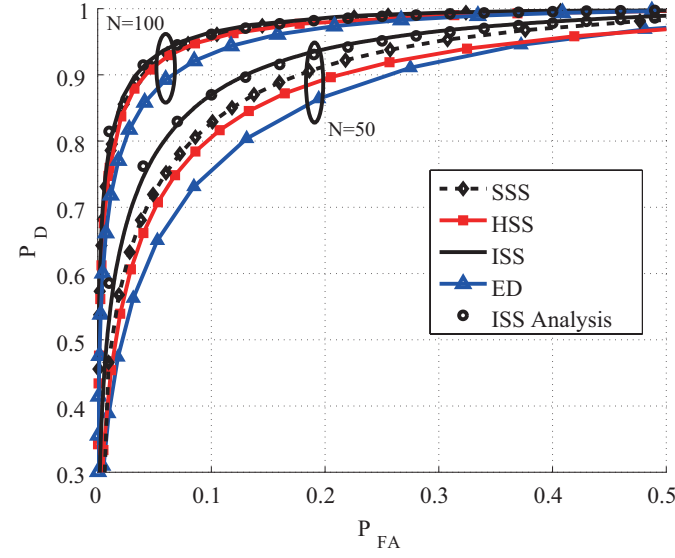

Fig. 1: Receiver operating characteristic curves of SSS, HSS, ISS, ISS (analysis), and ED. $\nu=-5 \mathrm{~dB}, N=50,100$, and $g_{0,0}=g_{1,1}=0.8$.

between results with $\nu=-5 \mathrm{~dB}$ and $\nu=-2 \mathrm{~dB}$ for all spectrum sensing scenarios indicates that the threshold is much more accurate in high $\nu$. We can also confirm that the gap between $P_{\mathrm{D}}$ of HSS and $P_{\mathrm{D}}$ of ISS decreases by increasing $N$. In fact, this behavior is not very straightforward since $\dot{P}_{\mathrm{D}}=$ 0.9 in HSS may cause constant error in $g_{\hat{x}_{t-1}, 0}$ of (8), but in ISS there is no error.

One reason for the above behavior is the difference between the two thresholds $\gamma_{0}^{(\mathrm{ED})}$ and $\gamma_{1}^{(\mathrm{ED})}$. To confirm this reason, achievable detection probabilities for each threshold as a function of $N$ are plotted in Fig. 3. The results are also obtained by Monte Carlo simulations. Increment of $N$ inherently enhances the sensing performance and the difference between the two thresholds decreases. Therefore, $P_{\mathrm{D}}$ of HSS also gets close to $\dot{P}_{\mathrm{D}}=0.9$ by increasing $N$.

In the region where $N<30$, both $P_{\mathrm{D}}$ and $P_{\mathrm{FA}}$ with threshold $\gamma_{1}^{(\mathrm{ED})}$ are high. In HSS, this fact leads to biased detection result $\hat{x}_{t}=1$, with $\gamma_{1}^{(\mathrm{ED})}$. Specifically, detection results of $\gamma_{1}^{(\mathrm{ED})}$ are $\hat{x}_{t}=1$ in most of cases under both $H_{0}$ and $H_{1}$. Therefore, $P_{\mathrm{D}}$ of HSS is high in the region $N<30$ in Fig. 2, and in fact $P_{\mathrm{FA}}$ of HSS is also significantly high.

\section{CONCLUSION}

Threshold setting for the spectrum sensing based on statistical information is investigated. The spectrum sensing based on statistical information can be expressed by ED with two thresholds. Analysis for the detection probability and false alarm probability of the spectrum sensing based on statistical information was obtained. This enables to set the threshold achieving the target detection probability. Numerical evaluations showed that the detection performance can be improved by exploiting the statistical information. We also showed that the analytical threshold setting is effective in case of a large number of samples for the spectrum sensing and a high signal to noise power ratio. In addition, a general form to set the thresholds in a general case in which more than two states model is assumed was also presented.

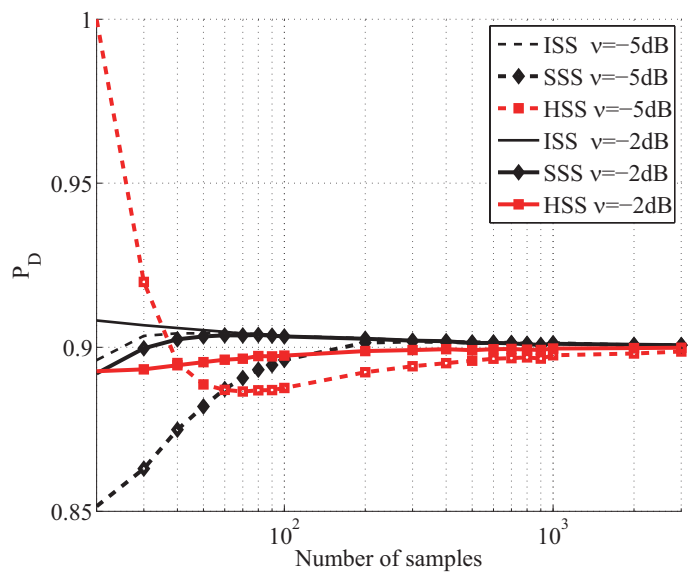

Fig. 2: $P_{\mathrm{D}}$ for SSS, HSS, and ISS as a function of the number of samples $N . \dot{P}_{\mathrm{D}}=0.9, \nu=-5 \mathrm{~dB}$ and $\nu=-2 \mathrm{~dB}$, and $g_{0,0}=g_{1,1}=0.8$.

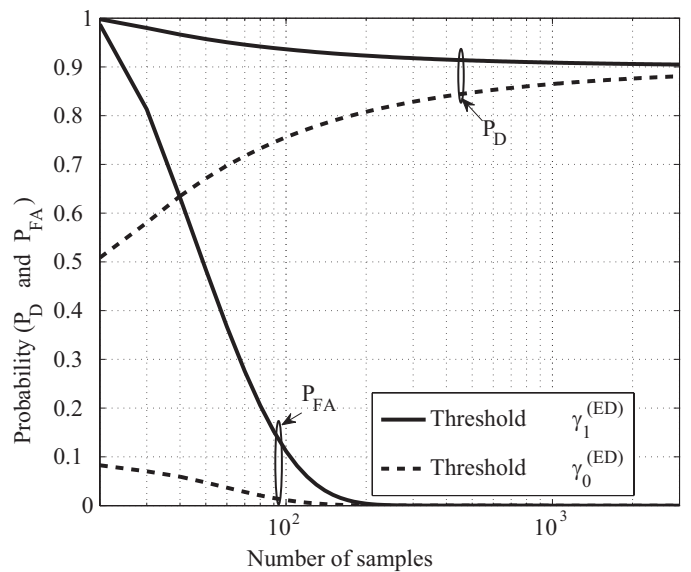

Fig. 3: $P_{\mathrm{D}}$ and $P_{\mathrm{FA}}$ for two thresholds $\gamma_{0}^{(\mathrm{ED})}\left(H_{0}\right)$ and $\gamma_{1}^{(\mathrm{ED})}$ $\left(H_{1}\right)$ as a function of the number of samples $N . \dot{P}_{\mathrm{D}}=0.9$, $\nu=-5 \mathrm{~dB}$, and $g_{0,0}=g_{1,1}=0.8$.

\section{REFERENCES}

[1] T. Yucek and H. Arslan, "A survey of spectrum sensing algorithms for cognitive radio applications," IEEE Communications Surveys \& Tutorials, vol. 11, pp. 116-130, Mar. 2009.

[2] N. Wang, Y. Gao, and X. Zhang, "Adaptive spectrum sensing algorithm under different primary user utilizations," IEEE Commun. Lett., vol. 17, no. 9, pp. 1838-1841, Sept. 2013.

[3] S. K. A. Mariani and A. Giorgetti, "Periodic spectrum sensing with noncontinuous primary user transmissions," IEEE Trans. Wireless Commun., pp. 1636-1949, Mar. 2015.

[4] T. Nguyen, B. L. Mark, and Y. Ephraim, "Spectrum sensing using a hidden bivariate markov model," IEEE Trans. Commun., pp. 4582-4591, Aug. 2013.

[5] H. Urkowitz, "Energy detection of unknown deterministic signals," Proc. IEEE, vol. 55, no. 4, pp. 523-531, Apr. 1967.

[6] L. E. Baum and T. Petrie, "Statistical inference for probabilistic functions of finite state markov chains," Ann. Math. Statist., vol. 37, pp. 1554-1563, Nov. 1966.

[7] M. López-Benítez and F. Casadevall, "Spectrum usage in cognitive radio networks: From field measurements to empirical models," IEICE Trans. Commun., vol. E97-B, no. 2, pp. 242-250, Feb. 2014.

[8] M. A. K. Abdulsattar and Z. A. Hussein, "Energy detector with baseband sampling for cognitive radio: Real-time implementation," Wireless Engineering and Technology, pp. 229-239, July 2012. 